\title{
Canonical elements for Öpik theory
}

\author{
Giacomo Tommei \\ Department of Mathematics, University of Pisa \\ via Buonarroti 2, 56127 Pisa, Italy \\ e-mail: tommei@dm.unipi.it
}

\begin{abstract}
The purpose of this paper is to find a set of canonical elements to use within the framework of Öpik theory of close encounters of a small body with a planet ([Öpik 1976]). Since the small body travels along a planetocentric hyperbola during the close approach and Öpik formulas are valid, without approximations, only at collision, we derive a set of canonical elements for hyperbolic collision orbits (eccentricity $e \rightarrow 1^{+}$, semi-major axis $a$ fixed) and then we introduce the unperturbed velocity of the small body and the distance covered along the asymptote as a new canonically conjugate pair of orbital elements. An interesting result would be to get a canonical set containing the coordinates in the Target Plane (TP), useful for the analysis of the future encounters: in the last part we prove that this is not possible.
\end{abstract}

Keywords:Öpik theory, hyperbolic collision orbits, canonical elements

\section{Introduction}

Öpik's theory of close encounters of a small body (asteroid, comet, meteoroid) with a planet is based on a 2-body approach ([Öpik 1976]). The small body is considered to be in a heliocentric ellipse until the time of the encounter with some planet, then, during the close approach, the dynamics is switched to a planetocentric hyperbolic orbit. Öpik's original expressions related the components of the planetocentric unperturbed velocity vector of the small body, $\mathbf{U}$, to the heliocentric orbital elements $a_{h}, e_{h}, i_{h}$. Strictly speaking, Öpik's formulas are valid only at collision. [Valsecchi et al. 2003] introduced corrections to first order in miss distance to extend them to close encounters. They also computed explicit expressions of $\xi$ and $\zeta$, the $b$-plane coordinates, from the heliocentric elements of the small body. The $b$-plane, or Target Plane (TP), is the plane containing the center of the planet and orthogonal to the incoming asymptote of the planetocentric hyperbola: more precisely this is the definition of the preencounter TP, while the post-encounter TP is perpendicular to the outgoing 
asymptote $^{1}$. [Valsecchi et al. 2003] introduced a non-canonical set of orbital elements $\left(U, \theta, \phi, \xi, \zeta, t_{0}\right)$, which they used to analyze the close approach and the dynamics generated. Here $U=|\mathbf{U}|$ is the planetocentric unperturbed velocity, $\theta$ and $\phi$ are the angles which define the direction of $\mathbf{U}$ in a planetocentric reference system $(X, Y, Z)$, where the $Y$-axis coincides with the direction of motion of the planet, and the Sun is on the negative $X$-axis; $t_{0}$ is the time of crossing of the ecliptic plane by the small body. These elements are not canonical. In this paper we derive a canonical set of elements to describe the dynamics of a small body during a planetary close encounter: we look for elements that remain well defined at collision. [Tremaine 2001] obtained a set of canonical elements for elliptic collision orbits (eccentricity $e \rightarrow 1^{-}$, semi-major axis $a$ fixed), using a couple of angles which define the direction of the vector pointing toward the apocenter of the orbit. Following that work we get (Section 2) a set of canonical elements for hyperbolic collision orbits $\left(e \rightarrow 1^{+}, a\right.$ fixed), using the angles which define the direction of the vector from the focus to the center of the planetocentric hyperbola. Then, we show that it is possible to keep a canonical set replacing the canonically conjugate couple $(L, l)$ of elements with the conjugate couple $(U, \eta)$, where $\eta$ is the distance covered by the small body along the asymptote, starting from a reference time. The position of the small body in the TP gives information about the encounter and the next close approaches, so it would be useful to obtain a canonical set containing some coordinates in that plane. In Section 3 we prove that it is not possible.

\section{Canonical hyperbolic collision elements}

The orbit of the small body during a planetary encounter (see Figure 1) is essentially a branch of a hyperbola with the planet at the relative focus. If $a$ is the distance of the pericenter $(\mathrm{PE})$ from the center of hyperbola $(\mathrm{C})$, and $a e$ is the distance between the focus $(\mathrm{O})$ and the center, the distance of the small body from the planet is

$$
r=\frac{a\left(e^{2}-1\right)}{1+e \cos f},
$$

where $f$ is the true anomaly.

There are few papers in literature dealing with the hyperbolic orbital motion analytically. As a starting point for our work we are interested in a set of canonical elements for hyperbolic orbits. [Hori 1961] modified the classical set of Delaunay obtaining elements applicable to hyperbolic orbital motion. [Floria 1995] derived the same set of elements defining a canonical transformation starting from Hill variables. We call that canonical set of elements the Delaunay hyperbolic elements $\left(\mathcal{D}_{\text {hyp }}\right)$ and we use this set as the basis of our deductions:

$$
\mathcal{D}_{\text {hyp }}=(L, G, H, l, g, h)
$$

\footnotetext{
${ }^{1}$ Hereafter when we shall speak of TP-coordinates we shall mean the pre-encounter TP: we are going to analyze the effects of the encounter in Subsection 2.1
} 


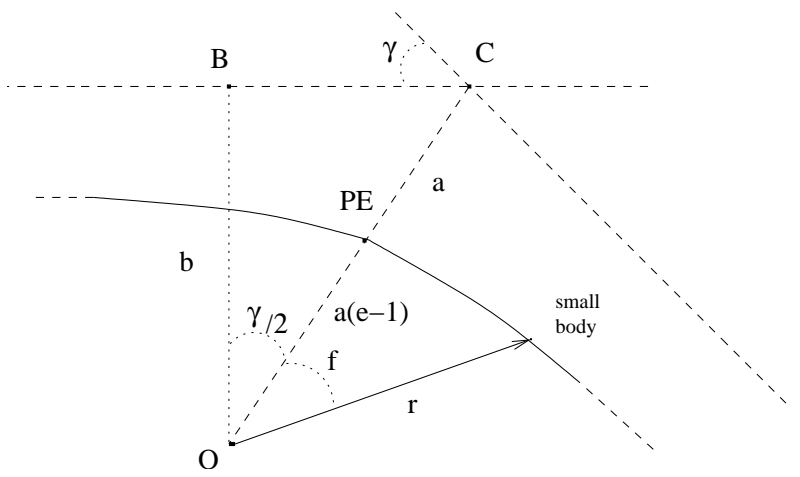

Figure 1: Planetocentric hyperbola with the focus at $\mathrm{O}$ and the center at $\mathrm{C}$. Point $\mathrm{PE}$ denotes the pericenter having distance $a$ from $\mathrm{C}$ and $a(e-1)$ from $\mathrm{O}$. The impact parameter $b$ is the distance from the focus to the incoming asymptote, which is equal to the distance from the focus to the outgoing asymptote. Angle $\gamma$ is the deflection angle, that is the angle between the asymptotes.

where

$$
\begin{aligned}
L & =-(\mu a)^{1 / 2} & & l=e \sinh F-F=n t+\text { const. } \\
G & =\left[\mu a\left(e^{2}-1\right)\right]^{1 / 2} & & g=\omega \\
H & =G \cos i & & h=\Omega
\end{aligned}
$$

In the previous relations, $(a, e, i, \omega, \Omega)$ are the usual Keplerian elements for a hyperbolic orbit, $\mu$ is the mass of the planet in units where the gravitational constant is unity, while $F$ is the hyperbolic eccentric anomaly defined by

$$
\cosh F=\frac{a+r}{a e} .
$$

The Hamiltonian of the 2-body problem becomes

$$
\mathcal{K}_{D_{\text {hyp }}}=\frac{\mu^{2}}{2 L^{2}} .
$$

We shall indicate with $\mathcal{D}_{\text {hyp }}^{5}$ the five elements $(L, G, H, g, h)$ which define the geometry of the orbit.

Now let us consider the unit sphere as shown in Figure 2. The orbital plane of the small body intersects the sphere along a great circle $D$. The ascending node is marked with $N$. The angular distance $A N$ is the longitude of the node $h$, and the inclination of the orbit is denoted by $i$. The versor $\hat{\mathbf{c}}=\left(\cos \theta_{C} \cos \phi_{C}, \cos \theta_{C} \sin \phi_{C}, \sin \theta_{C}\right)$, specified by the polar coordinates $\left(\theta_{C}, \phi_{C}\right)$, defines the direction from the planet to the center of the hyperbola. This direction remains well defined for collision orbits $\left(e \rightarrow 1^{+}, a\right.$ fixed) and thus the idea is to use the angles $\left(\theta_{C}, \phi_{C}\right)$ as canonical coordinates. 


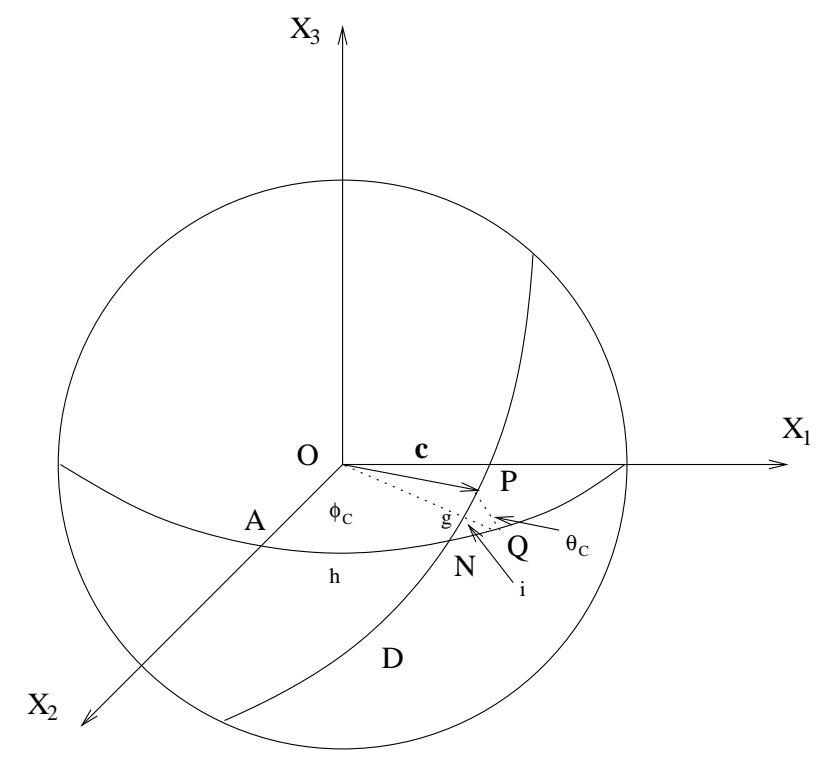

Figure 2: Configuration of the 2-body problem projected onto the unit sphere. The planet is at the origin of the ecliptic reference frame $\left(X_{1}, X_{2}, X_{3}\right)$. The versor $\hat{\mathbf{c}}$ denotes the direction from the focus to the center of the planetocentric hyperbola, that coincides with the direction of the pericenter when this is defined.

Following the work of [Tremaine 2001] we prove that

$$
\mathcal{C}_{\text {hyp }}=\left(L, \Theta, H, l, \theta_{C}, \phi_{C}\right)
$$

is a set of canonical elements.

The angles $\left(\theta_{C}, \phi_{C}\right)$ satisfy the following relations

$$
\begin{aligned}
& \sin \theta_{C}=\sin g \sin i \\
& \cos \theta_{C} \sin \left(\phi_{C}-h\right)=\sin g \cos i \\
& \cos \theta_{C} \cos \left(\phi_{C}-h\right)=\cos g
\end{aligned}
$$

which could be derived from usual theorems of spherical trigonometry applied to the spherical triangle $P N Q$ (Figure 3).

The momentum conjugate to the coordinate $\theta_{C}$ is the component of the angular momentum $\mathbf{G}$ along a line defined by the versor

$$
\hat{\mathbf{t}}=\left(\hat{X}_{3} \times \hat{\mathbf{c}}\right) / \cos \theta_{C}=\left(-\sin \phi_{C}, \cos \phi_{C}, 0\right)
$$

and we indicate it with $\Theta=\mathbf{G} \cdot \hat{\mathbf{t}}$.

Let us consider a canonical transformation from the set $\mathcal{D}_{\text {hyp }}$ to new momenta and coordinates $(\mathbf{I}, \mathbf{w})$ defined by the following generating function

$$
S(L, G, H, \mathbf{w})=-w_{1} L+\left(\frac{\pi}{2}-w_{3}\right) H-\frac{\pi}{2} G
$$




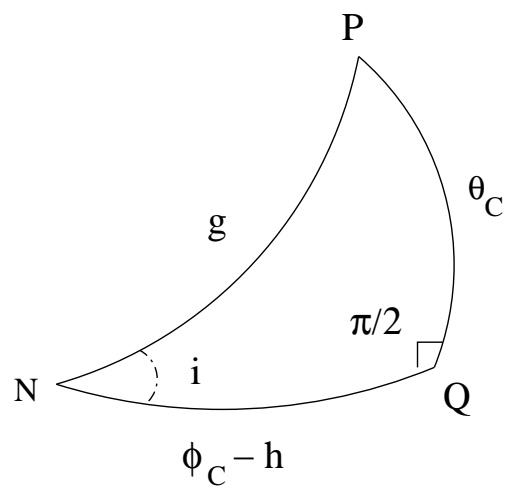

Figure 3: Spherical rectangular triangle $P N Q$. Point $P$ is the intersection of the versor $\hat{\mathbf{c}}$ with the sphere, while $N$ is the ascending node.

$$
\mp G \arccos \left[\frac{G \sin w_{2}}{\left(G^{2}-H^{2}\right)^{1 / 2}}\right] \pm H \arccos \left[\frac{H \tan w_{2}}{\left(G^{2}-H^{2}\right)^{1 / 2}}\right]
$$

where $0 \leq w_{2} \leq \pi$ and $0 \leq w_{3} \leq 2 \pi$. The arguments of the inverse trigonometric functions could also be expressed as

$$
\begin{gathered}
\frac{G \sin w_{2}}{\left(G^{2}-H^{2}\right)^{1 / 2}}=\frac{\sin w_{2}}{\sin i} \\
\frac{H \tan w_{2}}{\left(G^{2}-H^{2}\right)^{1 / 2}}=\frac{\tan w_{2}}{\tan i},
\end{gathered}
$$

so they have absolute value less than 1 if

$$
\sin w_{2}<|\sin i|
$$

that is, $w_{2} \in(0,|i|) \cup(\pi-|i|, \pi)$, considering $-\pi / 2 \leq i \leq \pi / 2, \quad i \neq 0$. Then,

$$
I_{1}=-\frac{\partial S}{\partial w_{1}}=L \quad I_{2}=-\frac{\partial S}{\partial w_{2}}=\mp\left(G^{2}-\frac{H^{2}}{\cos ^{2} w_{2}}\right)^{1 / 2} \quad I_{3}=-\frac{\partial S}{\partial w_{3}}=H
$$

and

$$
\begin{aligned}
l & =-\frac{\partial S}{\partial L}=w_{1} \\
g & =-\frac{\partial S}{\partial G}=\frac{\pi}{2} \pm \arccos \left(\frac{\sin w_{2}}{\sin i}\right) \\
h & =-\frac{\partial S}{\partial H}=w_{3}-\frac{\pi}{2} \mp \arccos \left(\frac{\tan w_{2}}{\tan i}\right)
\end{aligned}
$$

Using relations (3) and from simple computations we obtain

$$
\begin{aligned}
& \sin w_{2}=\sin g \sin i \\
& \cos w_{2} \sin \left(w_{3}-h\right)=\sin g \cos i \\
& \cos w_{2} \cos \left(w_{3}-h\right)=\cos g
\end{aligned}
$$


Comparing equations (4) with equations (2), we deduce

$$
w_{2}=\theta_{C} \quad w_{3}=\phi_{C} .
$$

The identity $I_{2}=\Theta$ is proved in Appendix A.1.

\subsection{Replacing the conjugate couple $(L, l)$ with $(U, \eta)$}

The consequence of the encounter of the small body with the planet is that vector $\mathbf{U}$, aligned with the incoming asymptote, is rotated into $\mathbf{U}^{\prime}$, aligned with the outgoing asymptote, while its magnitude does not change, that is $|\mathbf{U}|=\left|\mathbf{U}^{\prime}\right|=$ $U$. The deflection angle $\gamma$ (see [Carusi et al. 1990], Par.3; [Öpik 1976], Chap. 2, Par. 2.3; [Valsecchi et al. 2003], Par. 2.3) depends on the impact parameter $b$ (the distance $\mathrm{OB}$ in Figure 1) and on $U$. It is related to the geometry of the orbit through the expression

$$
\sin \frac{\gamma}{2}=\frac{1}{e}
$$

where $e$ is the eccentricity.

The velocity $U$ is related to the planetocentric semi-major axis by the relation ([Öpik 1976], Chap. 2, Par. 2.3, Formula [83])

$$
U=\left(\frac{\mu}{a}\right)^{1 / 2} .
$$

We shall prove that the set

$$
\mathcal{C}_{\text {opik }}=\left(U, \Theta, H, \eta, \theta_{C}, \phi_{C}\right)
$$

is canonical, where

$$
\eta=U\left(t-t_{0}\right)
$$

is the distance covered along the asymptote, starting from a reference time $t_{0}$ and considering the small body moving with constant velocity. Quantity $U$ is a function only depending on the hyperbolic Delaunay element $L$ (from relations (1) and (5)), while $\eta$ depends on $L$ and $l$ (from (1),(5) and (6)) :

$$
U=U(L)=-\frac{\mu}{L} \quad \eta=\eta(L, l)=\frac{L^{2} l}{\mu}
$$

The other relations between $\mathcal{C}_{\text {opik }}$ elements and $\mathcal{D}_{\text {hyp }}$ elements are in Appendix A.2. The transformation from $\mathcal{C}_{\text {hyp }}$ to $\mathcal{C}_{\text {opik }}$ is time-independent and it is completely canonical if and only if the Jacobian matrix

$$
\mathbf{F}=\frac{\partial \mathcal{C}_{\text {opik }}}{\partial \mathcal{C}_{\text {hyp }}}
$$

is symplectic, that is

$$
\mathbf{F}^{T} \mathbf{J} \mathbf{F}=\mathbf{J}
$$


where

$$
\mathbf{J}=\left[\begin{array}{cc}
\mathbf{0}_{3} & -\mathbf{I}_{3} \\
\mathbf{I}_{3} & \mathbf{0}_{3}
\end{array}\right]
$$

$\mathbf{F}$ has the form

$$
\mathbf{F}=\left[\begin{array}{ll}
\mathbf{A} & \mathbf{0} \\
\mathbf{C} & \mathbf{B}
\end{array}\right]
$$

with

$$
\mathbf{A}=\left[\begin{array}{ccc}
\frac{\partial U}{\partial L} & 0 & 0 \\
0 & 1 & 0 \\
0 & 0 & 1
\end{array}\right] \mathbf{B}=\left[\begin{array}{ccc}
\frac{\partial \eta}{\partial l} & 0 & 0 \\
0 & 1 & 0 \\
0 & 0 & 1
\end{array}\right] \mathbf{C}=\left[\begin{array}{ccc}
\frac{\partial \eta}{\partial L} & 0 & 0 \\
0 & 0 & 0 \\
0 & 0 & 0
\end{array}\right]
$$

and this implies

$$
\mathbf{F}^{T} \mathbf{J} \mathbf{F}=\left[\begin{array}{cc}
\mathbf{0} & -\mathbf{A} \mathbf{B} \\
\mathbf{A} \mathbf{B} & \mathbf{0}
\end{array}\right]
$$

Hence matrix $\mathbf{F}$ is symplectic if and only if

$$
\mathbf{A} \mathbf{B}=\mathbf{I}_{3},
$$

that is, if and only if

$$
\frac{\partial U}{\partial L} \frac{\partial \eta}{\partial l}=1
$$

Condition (7) is indeed satisfied, since

$$
\frac{\partial U}{\partial L}=\frac{\mu}{L^{2}} \quad \text { and } \quad \frac{\partial \eta}{\partial l}=\frac{L^{2}}{\mu} .
$$

The standard Keplerian Hamiltonian becomes

$$
\mathcal{K}_{C_{\text {opik }}}=\mathcal{K}_{C_{\text {opik }}}(U)=\frac{1}{2} U^{2},
$$

and the canonical equation of motion for the coordinate $\eta$ gives its conjugate momentum $U$

$$
\dot{\eta}=\frac{\partial \mathcal{K}}{\partial U}=U .
$$

The introduction of this set of elements gives prominence to the local behavior of the small body: around the time of crossing the pre-encounter TP, the small body travels with constant velocity $U$ along a straight line having the direction of the incoming asymptote; of course around the time of crossing the postencounter TP, the small body moves on a straight line having the direction of the outgoing asymptote.

Let us analyze the effect of the encounter considering the transformation that maps the pre-encounter state vector with components $\left(U, \Theta, H, \eta, \theta_{C}, \phi_{C}\right)$ 
onto the post-encounter state vector with components $\left(U^{\prime}, \Theta^{\prime}, H^{\prime}, \eta^{\prime}, \theta_{C}^{\prime}, \phi_{C}^{\prime}\right)$ :

$$
\begin{aligned}
U^{\prime} & =U \\
\Theta^{\prime} & =\Theta \\
H^{\prime} & =H \\
\eta^{\prime} & =\eta+U\left(t_{2}-t_{1}\right) \\
\theta_{C}^{\prime} & =\theta_{C} \\
\phi_{C}^{\prime} & =\phi_{C}
\end{aligned}
$$

where $t_{1}$ is the time of crossing the pre-encounter TP, while $t_{2}$ is the time of crossing the post-encounter TP (note that $t_{2}=t_{1}$ in [Valsecchi et al. 2003]). The 2-body propagation, like in ordinary treatment of Keplerian motion, is described by five constants and a time-dependent variable. This peculiarity makes this set less interesting to study the dynamics of the future close approaches, in particular the structure of resonance and keyholes ${ }^{2}$ ([Valsecchi et al. 2003]). Then a significant result would be to find a canonical set containing information about the position of the small body in the TP: this issue will be discussed in the next Section.

\section{Non-existence of canonical elements contain- ing TP coordinates}

The TP analysis is a powerful tool to analyze the dynamics of the close approaches. In order to characterize the position of the small body in the TP we fix two orthogonal axes, $\mathcal{X}$ and $\mathcal{Z}$, intersecting at the center of the planet. There are many ways to choose these axes: it is possible to align the $\mathcal{Z}$-axis with the projection of the normal to the ecliptic or in the direction opposite to the projection of the heliocentric velocity of the planet, as within the framework of Öpik theory. [Valsecchi et al. 2003] computed the explicit expressions of the TP-coordinates $\xi$ and $\zeta$ from the heliocentric elements of the small body and they used the set of non-canonical elements $\left(U, \theta, \phi, \xi, \zeta, t_{0}\right)$, showing that these quantities, which characterize an encounter, can be linked to those of previous and next close approaches. The set $\left(U, \theta, \phi, \xi, \zeta, t_{0}\right)$ is not canonical and we derived a canonical one in Subsection 2.1. But, as explained, it would be interesting to find a canonical set having the necessary elements to perform a complete analysis of the current and future close encounters. The coordinates of the small body in the TP are certainly essential: in fact they give the possibility to compute the location, size and shape of the keyholes.

We now discuss the possibility to obtain a canonical set of elements containing information about the position of the small body in the TP. The method we apply makes use of the formalism of Poisson brackets, that we shall denote with $\{$,$\} . The Poisson brackets of two functions f$ and $g$ of canonical variables

\footnotetext{
${ }^{2}$ Keyholes are small regions in the TP such that, if the small body passes through one of them, an impact with the planet will occur at the next encounter.
} 
$(\mathbf{p}, \mathbf{q})$ are given by the expression (see for example [Goldstein 1980], Chap. 9, Par. 9.4)

$$
\{f, g\}_{(\mathbf{p}, \mathbf{q})}=\sum_{j=1}^{n}\left[\frac{\partial f}{\partial q_{j}} \frac{\partial g}{\partial p_{j}}-\frac{\partial f}{\partial p_{j}} \frac{\partial g}{\partial q_{j}}\right] .
$$

If the set of canonical elements we are looking for exists, then it is possible to find a canonical transformation from $\mathcal{D}_{\text {hyp }}$ elements to these new elements, and in particular the Poisson brackets of each couple of new coordinates should be zero. Obviously two of these new coordinates should represent the position of the small body in the TP, so they should depend only on $\mathcal{D}_{\text {hyp }}^{5}$ elements, while the third one should give the position of the small body along the orbit and it should also depend on $l$. Therefore we look for three functions $(\xi, \zeta, \eta)$, acting as the new coordinate-type canonical variables,

$$
\begin{aligned}
\xi & : \mathcal{D}_{h y p}^{5} \rightarrow \mathbf{R} \\
\zeta & : \mathcal{D}_{h y p}^{5} \rightarrow \mathbf{R} \\
\eta & : \mathcal{D}_{h y p} \rightarrow \mathbf{R}
\end{aligned}
$$

such that

$$
\{\xi, \zeta\}=0 \quad\{\xi, \eta\}=0 \quad\{\zeta, \eta\}=0
$$

and

$$
\xi^{2}+\zeta^{2}=\mathcal{R}^{2}(b)
$$

where $\mathcal{R}(b)$ is a rescaling function of $b$. The impact parameter can be expressed as a function of the $\mathcal{D}_{\text {hyp }}$ elements, using the angular momentum computed when the small body intersects the TP

$$
b=b(L, G)=\frac{G}{U}=\left(-\frac{L}{\mu}\right) G=-\frac{L G}{\mu}
$$

Proposition 1. There exist two functions

$$
\begin{array}{rll}
\xi & : & \mathcal{D}_{h y p}^{5} \rightarrow \mathbf{R} \\
\zeta & : & \mathcal{D}_{h y p}^{5} \rightarrow \mathbf{R}
\end{array}
$$

which characterize the position of the small body in the TP in some reference system such that

$$
\{\xi, \zeta\}=0
$$

Proof. Let us suppose that we have chosen a reference system in the TP: then each point can be characterized by two coordinates $\xi$ and $\zeta$ depending on the $\mathcal{D}_{\text {hyp }}^{5}$ elements (in Appendix B we show how to compute some coordinates in the $\mathrm{TP}$ as functions of $\mathcal{D}_{\text {hyp }}^{5}$ elements). The distance, measured in the TP, of the small body from the origin of the reference system is $\mathcal{R}(b)$. Using this function of the impact parameter and a polar angle

$$
\psi \quad: \quad \mathcal{D}_{h y p}^{5} \rightarrow \mathbf{R},
$$


$\xi$ and $\zeta$ could be expressed as

$$
\left\{\begin{array}{l}
\xi=\mathcal{R}(b) \cos \psi \\
\zeta=\mathcal{R}(b) \sin \psi
\end{array}\right.
$$

If $\{\xi, \zeta\}=0$ we have just found the coordinates we are looking for. Otherwise let $\mathbf{R}_{\rho}$ be the rotation in the plane of an angle

$$
\begin{gathered}
\rho: \mathcal{D}_{\text {hyp }}^{5} \rightarrow \mathbf{R} \\
\mathbf{R}_{\rho}=\left[\begin{array}{cr}
\cos \rho & -\sin \rho \\
\sin \rho & \cos \rho
\end{array}\right]
\end{gathered}
$$

and let us apply it to our coordinates in the TP

$$
\left[\begin{array}{l}
\xi^{\prime} \\
\zeta^{\prime}
\end{array}\right]=\mathbf{R}_{\rho}\left[\begin{array}{l}
\xi \\
\zeta
\end{array}\right]=\left[\begin{array}{l}
\xi \cos \rho-\zeta \sin \rho \\
\xi \sin \rho+\zeta \cos \rho
\end{array}\right] .
$$

Before going on, we have to note that the transformation applied to $\xi$ and $\zeta$ is not a real rotation because the angle is not constant, but depends on the $\mathcal{D}_{\text {hyp }}^{5}$ elements. Using a non-canonical transformation is the only way to try to obtain canonical coordinates from two functions $\xi$ and $\zeta$ such that $\{\xi, \zeta\} \neq 0$.

Let us consider the Poisson brackets of the transformed coordinates. Starting from the result of the computations described in Appendix $\mathrm{C}$ and using the properties of the Poisson brackets we derive

$$
\begin{aligned}
\left\{\xi^{\prime}, \zeta^{\prime}\right\} & =\{\xi, \zeta\}+\xi\{\xi, \rho\}+\zeta\{\zeta, \rho\} \\
& =\{\xi, \zeta\}-[\xi\{\rho, \xi\}+\zeta\{\rho, \zeta\}] \\
& =\{\xi, \zeta\}-\frac{1}{2}\left[\left\{\rho, \xi^{2}\right\}+\left\{\rho, \zeta^{2}\right\}\right] \\
& =\{\xi, \zeta\}-\frac{1}{2}\left\{\rho, \xi^{2}+\zeta^{2}\right\} \\
& =\{\xi, \zeta\}-\frac{1}{2}\left\{\rho, \mathcal{R}^{2}(b)\right\} \\
& =\{\xi, \zeta\}-\mathcal{R}(b)\{\rho, \mathcal{R}(b)\} .
\end{aligned}
$$

Therefore, using relations (12) we have

$$
\begin{aligned}
\left\{\xi^{\prime}, \zeta^{\prime}\right\}=0 & \Leftrightarrow \mathcal{R}(b)\{\rho, \mathcal{R}(b)\}=\{\xi, \zeta\} \\
& \Leftrightarrow \mathcal{R}(b) \frac{\partial \rho}{\partial g} \frac{\partial \mathcal{R}(b)}{\partial G}=\{\xi, \zeta\},
\end{aligned}
$$

but, using (10)

$$
\{\xi, \zeta\}=-\mathcal{R}(b) \frac{\partial \mathcal{R}(b)}{\partial G} \frac{\partial \psi}{\partial g} .
$$

Then equation (13) is fulfilled if and only if

$$
\frac{\partial \rho}{\partial g}=-\frac{\partial \psi}{\partial g}
$$


that is

$$
\rho\left(\mathcal{D}_{\text {hyp }}^{5}\right)=-\psi\left(\mathcal{D}_{\text {hyp }}^{5}\right)+\psi_{0}(L, G, H, h),
$$

where $\psi_{0}(L, G, H, h)$ is a function which does not depend on $g$.

Applying the transformation (11) we get the set of coordinates required.

Remark 1. If $\xi$ and $\zeta$ are functions $\mathcal{D}_{\text {hyp }}^{5} \rightarrow \mathbf{R}$ representing the position of the small body in the TP, then

$$
\frac{\partial \xi}{\partial L} \neq 0 \quad \frac{\partial \zeta}{\partial L} \neq 0
$$

that is they depend on $L$.

This dependence follows from the definition of TP: it contains the impact parameter vector $\mathbf{b}$ which depends on the scattering angle $\gamma$, related to the $\mathcal{D}_{\text {hyp }}^{5}$ elements by

$$
\begin{aligned}
\sin \frac{\gamma}{2} & =\left(\frac{L^{2}}{L^{2}+G^{2}}\right)^{1 / 2} \\
\cos \frac{\gamma}{2} & =\left(\frac{G^{2}}{L^{2}+G^{2}}\right)^{1 / 2}
\end{aligned}
$$

and it is orthogonal to the unperturbed velocity $\mathbf{U}$; remember also that $U=|\mathbf{U}|$ depends on $L$,

$$
U=U(L)=-\frac{\mu}{L}
$$

Proposition 2. Let $\xi$ and $\zeta$ be two functions as in Proposition 1. Let us suppose that

$$
\eta\left(\mathcal{D}_{\text {hyp }}\right)=l^{N} \bar{\eta}\left(\mathcal{D}_{\text {hyp }}^{5}\right)+\widetilde{\eta}\left(\mathcal{D}_{\text {hyp }}^{5}\right), N \in \mathbf{Z}, \bar{\eta} \neq 0,
$$

where $l$ is the hyperbolic mean anomaly. Then $(\xi, \zeta, \eta)$ are not canonical coordinates.

Proof. Let us consider the Poisson brackets between $\xi$ and $\eta$

$$
\begin{aligned}
\{\xi, \eta\}= & -\frac{\partial \xi}{\partial L} \frac{\partial \eta}{\partial l}+\left(\frac{\partial \xi}{\partial g} \frac{\partial \eta}{\partial G}-\frac{\partial \xi}{\partial G} \frac{\partial \eta}{\partial g}\right)+\left(\frac{\partial \xi}{\partial h} \frac{\partial \eta}{\partial H}-\frac{\partial \xi}{\partial H} \frac{\partial \eta}{\partial h}\right) \\
= & -N l^{N-1} \bar{\eta} \frac{\partial \xi}{\partial L}+ \\
& l^{N}\left[\left(\frac{\partial \xi}{\partial g} \frac{\partial \bar{\eta}}{\partial G}-\frac{\partial \xi}{\partial G} \frac{\partial \bar{\eta}}{\partial g}\right)+\left(\frac{\partial \xi}{\partial h} \frac{\partial \bar{\eta}}{\partial H}-\frac{\partial \xi}{\partial H} \frac{\partial \bar{\eta}}{\partial h}\right)\right]+ \\
& {\left[\left(\frac{\partial \xi}{\partial g} \frac{\partial \widetilde{\eta}}{\partial G}-\frac{\partial \xi}{\partial G} \frac{\partial \widetilde{\eta}}{\partial g}\right)+\left(\frac{\partial \xi}{\partial h} \frac{\partial \widetilde{\eta}}{\partial H}-\frac{\partial \xi}{\partial H} \frac{\partial \widetilde{\eta}}{\partial h}\right)\right] } \\
= & -N l^{N-1} \bar{\eta} \frac{\partial \xi}{\partial L}+l^{N}\{\xi, \bar{\eta}\}+\{\xi, \widetilde{\eta}\}
\end{aligned}
$$


Then $\{\xi, \eta\}=0$ if and only if

$$
l^{N-1}\left[N \bar{\eta} \frac{\partial \xi}{\partial L}-l\{\xi, \bar{\eta}\}\right]=\{\xi, \widetilde{\eta}\}
$$

Since only the left-hand side depends on $l,\{\xi, \eta\}=0$ if and only if both sides are zero, that is

$$
\{\xi, \widetilde{\eta}\}=0
$$

and

$$
N \bar{\eta} \frac{\partial \xi}{\partial L}-l\{\xi, \bar{\eta}\}=0 .
$$

Equation (15) is equivalent to

$$
N \bar{\eta} \frac{\partial \xi}{\partial L}=l\{\xi, \bar{\eta}\},
$$

and, since only the right-hand side depends on $l$, it is fulfilled if and only if both sides are zero. But from the hypotheses and from Remark 1 the left-hand side cannot be zero. Consequently, $\{\xi, \eta\} \neq 0$. Reasoning in an analogous way one also concludes that $\{\zeta, \eta\} \neq 0$.

The choice of that particular form for the function $\eta$ of the previous Proposition will be clear during the proof of Theorem 1 .

Corollary 1. Let $\xi$ and $\zeta$ be two functions as in Proposition 1. If $\eta$ is the distance covered by the small body along the asymptote (the coordinate conjugate to the momentum $U)$, then $(\xi, \zeta, \eta)$ are not canonical coordinates.

Proof. Since

$$
\eta=\eta(L, l)=\frac{L^{2} l}{\mu}
$$

the thesis follows directly from Proposition 2, considering

$$
N=1 \quad \widetilde{\eta}=0 \quad \text { and } \quad \bar{\eta}=\frac{L^{2}}{\mu}
$$

Finally, we can prove the main result.

Theorem 1. If $\xi$ and $\zeta$ are two functions as in Proposition 1, then it is not possible to find a function

$$
\eta \quad: \quad \mathcal{D}_{\text {hyp }} \rightarrow \mathbf{R}
$$

such that

$$
\{\xi, \eta\}=0 \quad\{\zeta, \eta\}=0 .
$$


Proof. Proceeding by reductio ad absurdum, let us suppose that there exists a function

$$
\eta \quad: \quad \mathcal{D}_{h y p} \rightarrow \mathbf{R}
$$

such that

$$
\{\xi, \eta\}=0 \quad\{\zeta, \eta\}=0,
$$

where $\xi$ and $\zeta$ are as in Proposition 1. Then

$$
\begin{aligned}
& \xi\{\eta, \xi\}=\frac{1}{2}\left\{\eta, \xi^{2}\right\}=0 \\
& \zeta\{\eta, \zeta\}=\frac{1}{2}\left\{\eta, \zeta^{2}\right\}=0
\end{aligned}
$$

and, adding up these two previous equations, we obtain

$$
\frac{1}{2}\left\{\eta, \mathcal{R}^{2}(b)\right\}=0
$$

that is

$$
\frac{1}{2}\left[\frac{\partial \eta}{\partial l} \frac{\partial \mathcal{R}^{2}(b)}{\partial L}+\frac{\partial \eta}{\partial g} \frac{\partial \mathcal{R}^{2}(b)}{\partial G}\right]=0 .
$$

Equation (16) is fulfilled if and only if

$$
G \frac{\partial \eta}{\partial l}+L \frac{\partial \eta}{\partial g}=0
$$

The linear homogeneous partial differential equation (17) admits the family of solutions ${ }^{3}$

$$
\eta\left(\mathcal{D}_{\text {hyp }}\right)=\eta(L, G, H, \mathcal{Q}(L, G, l, g), h),
$$

where

$$
\mathcal{Q}(L, G, l, g)=L l-G g .
$$

Being $\mathcal{S}$ the family of solutions of (17), we have proved that

$$
\begin{aligned}
& \exists \eta\left(\mathcal{D}_{\text {hyp }}\right):\{\xi, \eta\}=0 \text { and }\{\zeta, \eta\}=0 \\
\Rightarrow & \eta\left(\mathcal{D}_{\text {hyp }}\right) \in \mathcal{S}
\end{aligned}
$$

but the following implication is also true

$$
\begin{aligned}
& \eta\left(\mathcal{D}_{\text {hyp }}\right) \notin \mathcal{S} \\
& \Rightarrow \quad \forall \eta\left(\mathcal{D}_{\text {hyp }}\right) \quad\{\xi, \eta\} \neq 0 \text { or }\{\zeta, \eta\} \neq 0 .
\end{aligned}
$$

Proving that if $\eta$ belongs to $\mathcal{S}$, then $\{\xi, \zeta, \eta\}$ cannot be canonical coordinates, we shall complete our proof.

\footnotetext{
${ }^{3}$ The general solution can be found with the method of characteristics
} 
Let us compute the Poisson brackets between $\xi$ and $\eta$, with $\eta \in \mathcal{S}$. Since $\eta$ has the form

$$
\eta\left(\mathcal{D}_{\text {hyp }}\right)=\eta(L, G, H, \mathcal{Q}(L, G, l, g), h),
$$

its partial derivatives are given by

$$
\begin{array}{rlrl}
\frac{\partial \eta}{\partial L} & =\left[\frac{\partial \eta}{\partial L}\right]+l \frac{\partial \eta}{\partial \mathcal{Q}}, & \frac{\partial \eta}{\partial l}=L \frac{\partial \eta}{\partial \mathcal{Q}}, \\
\frac{\partial \eta}{\partial G}=\left[\frac{\partial \eta}{\partial G}\right]-g \frac{\partial \eta}{\partial \mathcal{Q}}, & \frac{\partial \eta}{\partial g}=-G \frac{\partial \eta}{\partial \mathcal{Q}}, \\
\frac{\partial \eta}{\partial H}=\left[\frac{\partial \eta}{\partial H}\right], & \frac{\partial \eta}{\partial h}=\left[\frac{\partial \eta}{\partial h}\right],
\end{array}
$$

where the expressions in the square brackets are the "partial" partial derivatives of $\eta$ (we account for the contribution of terms occurring explicitly, leaving function $\mathcal{Q}$ aside), while the other partial derivatives (w.r.t. canonical variables in $\mathcal{D}_{\text {hyp }}$ ) are computed taking into account the explicit dependence plus terms generated via function $\mathcal{Q}$.

Given that $\xi$ does not depend on $l$, partial derivatives of $\eta$ with respect to $L$ are not significant while forming the Poisson brackets. Then

$$
\begin{aligned}
\{\xi, \eta\} & =-L \frac{\partial \xi}{\partial L} \frac{\partial \eta}{\partial \mathcal{Q}}+\left(\frac{\partial \xi}{\partial g} \frac{\partial \eta}{\partial G}+G \frac{\partial \xi}{\partial G} \frac{\partial \eta}{\partial \mathcal{Q}}\right)+\left(\frac{\partial \xi}{\partial h} \frac{\partial \eta}{\partial H}-\frac{\partial \xi}{\partial H} \frac{\partial \eta}{\partial h}\right) \\
& =\frac{\partial \eta}{\partial \mathcal{Q}}\left(G \frac{\partial \xi}{\partial G}-L \frac{\partial \xi}{\partial L}\right)+\frac{\partial \xi}{\partial g} \frac{\partial \eta}{\partial G}+\frac{\partial \xi}{\partial h} \frac{\partial \eta}{\partial H}-\frac{\partial \xi}{\partial H} \frac{\partial \eta}{\partial h}
\end{aligned}
$$

Therefore

$$
\{\xi, \eta\}=0
$$

if and only if

$$
\frac{\partial \eta}{\partial \mathcal{Q}}\left(L \frac{\partial \xi}{\partial L}-G \frac{\partial \xi}{\partial G}\right)=\frac{\partial \xi}{\partial g} \frac{\partial \eta}{\partial G}+\frac{\partial \xi}{\partial h} \frac{\partial \eta}{\partial H}-\frac{\partial \xi}{\partial H} \frac{\partial \eta}{\partial h}
$$

that is, if and only if

$$
\left(\frac{\partial \eta}{\partial \mathcal{Q}}\right)^{-1}\left(\frac{\partial \xi}{\partial g} \frac{\partial \eta}{\partial G}+\frac{\partial \xi}{\partial h} \frac{\partial \eta}{\partial H}-\frac{\partial \xi}{\partial H} \frac{\partial \eta}{\partial h}\right)
$$

does not depend on $l$.

If $\partial \eta / \partial \mathcal{Q}$ does not depend on $l$, then $\eta$ is a linear function of $\mathcal{Q}$ and it can be expressed in the form (14). From Proposition 2 we can conclude that $(\xi, \zeta, \eta)$ cannot be canonical coordinates.

If $\partial \eta / \partial \mathcal{Q}$ depends on the mean anomaly, expression (18) does not contain $l$ if and only if the second factor can be factorized as

$$
\frac{\partial \eta}{\partial \mathcal{Q}} \widehat{\eta}
$$


where

$$
\widehat{\eta}: \mathcal{D}_{h y p}^{5} \rightarrow \mathbf{R}
$$

is a function which does not depend on $l$.

Since that part is composed of a sum of three terms and each term is a product of a function of $\mathcal{D}_{\text {hyp }}$ elements and of a function of $\mathcal{D}_{\text {hyp }}^{5}$ elements, the factor $\partial \eta / \partial \mathcal{Q}$ must occur in each individual term. But this is impossible for derivatives with respect to $H$ and $h$, because $\mathcal{Q}$ does not depend on these elements. If $\eta$ does not depend on $H$ and $h$ we have

$$
\{\xi, \eta\}=0
$$

if and only if

$$
\frac{\partial \eta}{\partial \mathcal{Q}}\left(L \frac{\partial \xi}{\partial L}-G \frac{\partial \xi}{\partial G}\right)=\frac{\partial \xi}{\partial g} \frac{\partial \eta}{\partial G}
$$

that is

$$
\frac{\partial \eta}{\partial G}=\frac{\partial \eta}{\partial \mathcal{Q}} \check{\eta},
$$

where $\check{\eta}$ does not depend on $l$. Therefore $\eta$ depends on $G$ only through $\mathcal{Q}$ and the equation (19) is fulfilled if and only if $\xi$ satisfies the following linear homogeneous partial differential equation

$$
L \frac{\partial \xi}{\partial L}=(G-g) \frac{\partial \xi}{\partial G} .
$$

Equation (20) admits the family of solutions

$$
\xi=\xi(L(G-g), H, g, h),
$$

and, considering the Poisson brackets between $\zeta$ and $\eta$, we obtain an equivalent result, that is

$$
\zeta=\zeta(L(G-g), H, g, h) .
$$

But functions of the form (21) and (22) do not satisfy the relation (8) on the TP coordinates required by the hypotheses of the theorem. The sum of the squares of these two functions cannot contain $g$; if choosing some ad hoc functions this element disappears, then $L$ and $G$ also disappear, while $\mathcal{R}^{2}(b)$ depends on these elements. This contradiction completes our proof.

\section{Conclusions}

In order to obtain a set of canonical elements useful within the framework of Öpik theory, we have initially derived a canonical set for hyperbolic collision orbits $\left(\mathcal{C}_{\text {hyp }}\right)$, using two spherical polar angles which define the direction from the focus to the center of the planetocentric hyperbola. This direction remains welldefined for collision orbits and out of collision coincides with that of pericenter of the orbit. Subsequently we have replaced the canonically conjugate couple $(L, l)$ 
with the asymptotic velocity $U$ and the distance $\eta$ covered along the asymptote, obtaining a new set of canonical elements $\left(\mathcal{C}_{\text {opik }}\right)$, with a new Hamiltonian which expresses the local behavior of the small body around the time of crossing the TP.

The problem we have studied in the second part of the paper deals with the possibility to get canonical coordinates containing useful information to understand the dynamics generated by the encounter. Since the TP is a powerful tool for this aim, the original idea was to try to find a canonical set of elements containing some coordinates on the TP. But we have proved (Theorem 1) that this is not possible. Intuitively it can be explained by the fact that, if one of the three coordinates gives the position of the small body along the orbit (and therefore depends on the mean anomaly), the other two coordinates should supply the orientation of the orbit in the space, while TP-coordinates provide information about the shape of the orbit (they depend on semi-major axis and eccentricity).

Acknowledgements I wish to thank A. Milani, who proposed the problem, for his suggestions. I am grateful to G.F. Gronchi and G.B. Valsecchi for useful discussions on the subject of the paper and to P. Acquistapace for some suggestions for the last part. Particular thanks to L. Floría for the constructive comments contained in the detailed review.

\section{A Computational details}

\section{A.1 $I_{2}=\Theta$}

Let us show that $I_{2}=\Theta$. Momentum $I_{2}$ could be expressed as

$$
\begin{aligned}
I_{2} & =\mp\left(G^{2}-\frac{H^{2}}{\cos ^{2} \theta_{C}}\right)^{1 / 2}=\mp \frac{G}{\cos \theta_{C}}\left(\cos ^{2} \theta_{C}-\cos ^{2} i\right)^{1 / 2} \\
& =\mp \frac{G}{\cos \theta_{C}}\left(1-\sin ^{2} g \sin ^{2} i-\cos ^{2} i\right)^{1 / 2} \\
& =\mp \frac{G}{\cos \theta_{C}}\left(\sin ^{2} i-\sin ^{2} g \sin ^{2} i\right)^{1 / 2}=\mp \frac{G}{\cos \theta_{C}} \sin i \cos g
\end{aligned}
$$

while

$$
\Theta=\mathbf{G} \cdot \hat{\mathbf{t}}=\left(G_{X_{1}}, G_{X_{2}}, G_{X_{3}}\right) \cdot\left(-\sin \phi_{C}, \cos \phi_{C}, 0\right)
$$

where

$$
\begin{aligned}
G_{X_{1}} & = \pm G \sin i \sin h \\
G_{X_{2}} & =\mp G \sin i \cos h \\
G_{X_{3}} & =G \cos i
\end{aligned}
$$

and, by solving from equations (2),

$$
\begin{aligned}
\cos \phi_{C} & =\frac{\cos g \cos h-\sin g \sin h \cos i}{\cos \theta_{C}} \\
\sin \phi_{C} & =\frac{\sin g \cos h \cos i+\cos g \sin h}{\cos \theta_{C}}
\end{aligned}
$$


Therefore

$$
\Theta=\mathbf{G} \cdot \hat{\mathbf{t}}=-G_{X_{1}} \sin \phi_{C}+G_{X_{2}} \cos \phi_{C}=\mp \frac{G}{\cos \theta_{C}} \sin i \cos g=I_{2} .
$$

\section{A.2 $\mathcal{C}_{\text {opik }}$ elements from $\mathcal{D}_{\text {hyp }}$ elements}

Let us express the Öpik canonical elements as functions of Delaunay hyperbolic elements:

$$
\begin{aligned}
U & =-\frac{\mu}{L} \\
\Theta & =\mp \frac{G\left(G^{2}-H^{2}\right)^{1 / 2} \cos g}{\left(G^{2}-\left(G^{2}-H^{2}\right) \sin ^{2} g\right)^{1 / 2}} \\
H & =H \\
\eta & =\frac{L^{2} l}{\mu} \\
\cos \theta_{C} & =\frac{\left(G^{2}-\left(G^{2}-H^{2}\right) \sin ^{2} g\right)^{1 / 2}}{G} \\
\cos \phi_{C} & =\frac{G \cos g \cos h-H \sin g \sin h}{\left.\left(G^{2}-\left(G^{2}-H^{2}\right) \sin \right)^{2} g\right)^{1 / 2}} .
\end{aligned}
$$

\section{B Computation of some coordinates on TP as functions of $\mathcal{D}_{\text {hyp }}$ elements}

Let us start from Figure 4. Versor $\hat{\mathbf{c}}$ has the direction from the planet to the center of the hyperbola, while versor $\hat{\mathbf{b}}$ has the direction orthogonal to the incoming asymptote.

Notice that $\hat{\mathbf{b}}=\left(\cos \theta_{B} \cos \phi_{B}, \cos \theta_{B} \sin \phi_{B}, \sin \theta_{B}\right)$ is expressed by the polar coordinates $\left(\theta_{B}, \phi_{B}\right)$ which satisfy the following relations (see Figure 5 , spherical triangle $B N T)$

$$
\begin{aligned}
& \sin \theta_{B}=\sin \widetilde{g} \sin i \\
& \cos \theta_{B} \sin \widetilde{h}=\sin \widetilde{g} \cos i \\
& \cos \theta_{B} \cos \widetilde{h}=\cos \widetilde{g}
\end{aligned}
$$

where

$$
\widetilde{g}=g-\frac{\gamma}{2} \quad \text { and } \quad \widetilde{h}=\phi_{B}-h ;
$$

The deflection angle $\gamma$ could be expressed in terms of $\mathcal{D}_{\text {hyp }}^{5}$ elements:

$$
\begin{aligned}
\sin \frac{\gamma}{2} & =\left(\frac{L^{2}}{L^{2}+G^{2}}\right)^{1 / 2} \\
\cos \frac{\gamma}{2} & =\left(\frac{G^{2}}{L^{2}+G^{2}}\right)^{1 / 2}
\end{aligned}
$$

Since the TP contains $\hat{\mathbf{b}}$ and it is orthogonal to the incoming asymptote of the hyperbola, it intersects the unit sphere along a great circle: let us denote with $\psi$ the arc of this circle from the point $\mathrm{B}$ to the plane $X_{1} X_{2}$. Choosing the intersection of the TP with the plane $X_{1} X_{2}$ as the $\mathcal{X}$ axis, the coordinates of a point in the TP could 


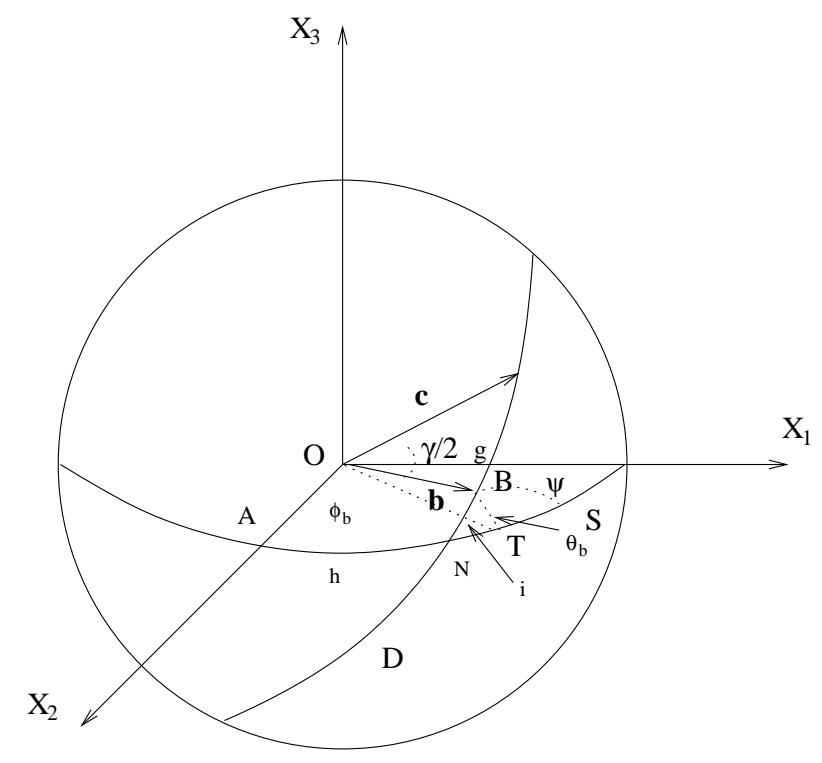

Figure 4: Configuration of the 2-body problem projected onto the unit sphere. We also depicted the direction of the impact parameter and the arc, denoted by $\psi$, of the great circle intersection of the TP with the sphere.

be expressed as in (10). The expression of the impact parameter $b=b(L, G)$ as a function of $\mathcal{D}_{\text {hyp }}^{5}$ elements is given by the relation (9), while in order to express $\cos \psi$ and $\sin \psi$ in terms of $\mathcal{D}_{\text {hyp }}^{5}$ elements we have to work on spherical triangles BTS and $B N T$ represented in Figure 5.

Let us denote with $\epsilon$ the angle $N \hat{B} T$. Working on BTS we obtain the relations

$$
\begin{aligned}
\cos \psi & =\cos \theta_{B} \cos T S \\
\cos T S & =\cos \theta_{B} \cos \psi+\sin \theta_{B} \sin \psi \sin \epsilon
\end{aligned}
$$

which combined with (see triangle $B N T$, Figure 5)

$$
\begin{aligned}
\frac{\sin \epsilon}{\sin \widetilde{h}} & =\frac{\sin i}{\sin \theta_{B}} \\
\sin \widetilde{h} & =\frac{\sin \widetilde{g} \cos i}{\cos \theta_{B}}
\end{aligned}
$$

give us

$$
\left\{\begin{array}{l}
\cos \psi= \pm \frac{\cos i}{\left(\sin ^{2} \theta_{B}+\cos ^{2} i\right)^{1 / 2}} \\
\sin \psi= \pm \frac{\sin \theta_{B}}{\left(\sin ^{2} \theta_{B}+\cos ^{2} i\right)^{1 / 2}}
\end{array}\right.
$$

Let us express these quantities in terms of $\mathcal{D}_{\text {hyp }}^{5}$ elements. The inclination is a function only of $G$ and $H$

$$
\cos i=\frac{H}{G}
$$




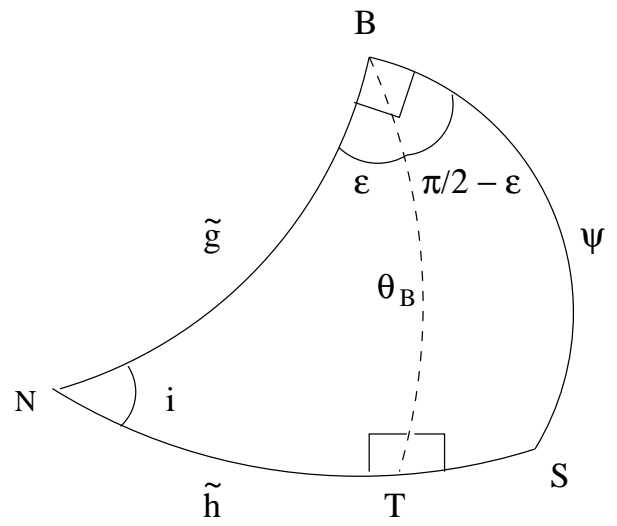

Figure 5: Spherical triangle $B N S$, union of the two spherical rectangular triangles $B N T$ and $B T S$. Point $B$ is the intersection of the versor $\hat{\mathbf{b}}$ with the sphere.

$$
\sin i= \pm\left(\frac{G^{2}-H^{2}}{G^{2}}\right)^{1 / 2}
$$

while $\theta_{B}$ also depends on $L$ (through the rotation of $\gamma / 2$ ) and $g$

$$
\begin{aligned}
\sin \theta_{B} & =\sin \widetilde{g} \sin i \\
& =\left(\sin g \cos \frac{\gamma}{2}-\sin \frac{\gamma}{2} \cos g\right) \sin i \\
& = \pm(G \sin g-L \cos g)\left[\frac{G^{2}-H^{2}}{G^{2}\left(L^{2}+G^{2}\right)}\right]^{1 / 2}
\end{aligned}
$$

Now we can express $\xi$ and $\zeta$ up to a sign:

$$
\begin{aligned}
& \xi= \pm\left[-\frac{L G}{\mu}\right]\left[\frac{\alpha}{\alpha+\beta}\right]^{1 / 2} \\
& \zeta= \pm\left[-\frac{L G}{\mu}\right]\left[\frac{\beta}{\alpha+\beta}\right]^{1 / 2}
\end{aligned}
$$

where

$$
\begin{aligned}
\alpha & =\alpha(L, G, H)=H^{2}\left(L^{2}+G^{2}\right) \\
\beta & =\beta(L, G, H, g)=\left(G^{2}-H^{2}\right)(G \sin g-L \cos g)^{2}
\end{aligned}
$$

\section{Poisson brackets of the transformed coordi- nates}

Let us express the Poisson brackets of the coordinates $\xi^{\prime}\left(\mathcal{D}_{\text {hyp }}^{5}\right)$ and $\zeta^{\prime}\left(\mathcal{D}_{\text {hyp }}^{5}\right)$ obtained with the transformation (11). 
First of all let us compute the derivatives.

$$
\begin{aligned}
\frac{\partial \xi^{\prime}}{\partial g} & =\left(\frac{\partial \xi}{\partial g} \cos \rho-\frac{\partial \zeta}{\partial g} \sin \rho\right)-\frac{\partial \rho}{\partial g}(\xi \sin \rho+\zeta \cos \rho)=\mathcal{A}-\frac{\partial \rho}{\partial g} \zeta^{\prime} \\
\frac{\partial \zeta^{\prime}}{\partial G} & =\left(\frac{\partial \xi}{\partial G} \sin \rho+\frac{\partial \zeta}{\partial G} \cos \rho\right)+\frac{\partial \rho}{\partial G}(\xi \cos \rho-\zeta \sin \rho)=\mathcal{B}+\frac{\partial \rho}{\partial G} \xi^{\prime} \\
\frac{\partial \xi^{\prime}}{\partial G} & =\left(\frac{\partial \xi}{\partial G} \cos \rho-\frac{\partial \zeta}{\partial G} \sin \rho\right)-\frac{\partial \rho}{\partial G}(\xi \sin \rho+\zeta \cos \rho)=\mathcal{C}-\frac{\partial \rho}{\partial G} \zeta^{\prime} \\
\frac{\partial \zeta^{\prime}}{\partial g} & =\left(\frac{\partial \xi}{\partial g} \sin \rho+\frac{\partial \zeta}{\partial g} \cos \rho\right)+\frac{\partial \rho}{\partial g}(\xi \cos \rho-\zeta \sin \rho)=\mathcal{D}+\frac{\partial \rho}{\partial g} \xi^{\prime} \\
\frac{\partial \xi^{\prime}}{\partial h} & =\left(\frac{\partial \xi}{\partial h} \cos \rho-\frac{\partial \zeta}{\partial h} \sin \rho\right)-\frac{\partial \rho}{\partial h}(\xi \sin \rho+\zeta \cos \rho)=\mathcal{E}-\frac{\partial \rho}{\partial h} \zeta^{\prime} \\
\frac{\partial \zeta^{\prime}}{\partial H} & =\left(\frac{\partial \xi}{\partial H} \sin \rho+\frac{\partial \zeta}{\partial H} \cos \rho\right)+\frac{\partial \rho}{\partial H}(\xi \cos \rho-\zeta \sin \rho)=\mathcal{F}+\frac{\partial \rho}{\partial H} \xi^{\prime} \\
\frac{\partial \xi^{\prime}}{\partial H} & =\left(\frac{\partial \xi}{\partial H} \cos \rho-\frac{\partial \zeta}{\partial H} \sin \rho\right)-\frac{\partial \rho}{\partial H}(\xi \sin \rho+\zeta \cos \rho)=\mathcal{M}-\frac{\partial \rho}{\partial H} \zeta^{\prime} \\
\frac{\partial \zeta^{\prime}}{\partial h} & =\left(\frac{\partial \xi}{\partial h} \sin \rho+\frac{\partial \zeta}{\partial h} \cos \rho\right)+\frac{\partial \rho}{\partial h}(\xi \cos \rho-\zeta \sin \rho)=\mathcal{N}+\frac{\partial \rho}{\partial h} \xi^{\prime}
\end{aligned}
$$

From previous relations we have

$$
\begin{aligned}
\left\{\xi^{\prime}, \zeta^{\prime}\right\}= & (\mathcal{A B}-\mathcal{C} \mathcal{D})+\frac{\partial \rho}{\partial G}\left(\mathcal{A} \xi^{\prime}+\mathcal{D} \zeta^{\prime}\right)-\frac{\partial \rho}{\partial g}\left(\mathcal{B} \zeta^{\prime}+\mathcal{C} \xi^{\prime}\right)+ \\
& (\mathcal{E} \mathcal{F}-\mathcal{M} \mathcal{N})+\frac{\partial \rho}{\partial H}\left(\mathcal{E} \xi^{\prime}+\mathcal{N} \zeta^{\prime}\right)-\frac{\partial \rho}{\partial h}\left(\mathcal{F} \zeta^{\prime}+\mathcal{M} \xi^{\prime}\right),
\end{aligned}
$$

and from more computations

$$
\begin{aligned}
& (\mathcal{A B}-\mathcal{C} \mathcal{D})+(\mathcal{E} \mathcal{F}-\mathcal{M} \mathcal{N})=\{\xi, \zeta\} \\
& \frac{\partial \rho}{\partial G}\left(\mathcal{A} \xi^{\prime}+\mathcal{D} \zeta^{\prime}\right)-\frac{\partial \rho}{\partial g}\left(\mathcal{B} \zeta^{\prime}+\mathcal{C} \xi^{\prime}\right)+ \\
& \frac{\partial \rho}{\partial H}\left(\mathcal{E} \xi^{\prime}+\mathcal{N} \zeta^{\prime}\right)-\frac{\partial \rho}{\partial h}\left(\mathcal{F} \zeta^{\prime}+\mathcal{M} \xi^{\prime}\right)=\xi\{\xi, \rho\}+\zeta\{\zeta, \rho\} .
\end{aligned}
$$

Therefore

$$
\left\{\xi^{\prime}, \zeta^{\prime}\right\}=\{\xi, \zeta\}+\xi\{\xi, \rho\}+\zeta\{\zeta, \rho\}
$$

\section{References}

[Carusi et al. 1990] Carusi A., Valsecchi G.B. and Greenberg R. : 1990 Planetary close encounters: geometry of approach and post-encounter orbital parameters, Celestial Mechanics and Dynamical Astronomy, Volume 49, Issue 2, pp. 111-131

[Floria 1995] Floria L. : 1995 A simple derivation of the hyperbolic Delaunay variables, The Astronomical Journal, Volume 110, Number 2, pp. 940-942

[Goldstein 1980] Goldstein H. : 1980 Classical Mechanics, Second Edition, Addison Wesley

[Hori 1961] Hori G. : 1961 The Motion of a Hyperbolic Artificial Satellite around the Oblate Earth, The Astronomical Journal, Volume 66, Number 6, pp. 258-263 
[Öpik 1976] Öpik E.J. : 1976 Interplanetary Encounters, Elsevier, AmsterdamOxford-New York

[Tremaine 2001] Tremaine, S. : 2001 Canonical elements for collision orbits, Celestial Mechanics and Dynamical Astronomy, Volume 79, Issue 3, pp. 231-233

[Valsecchi et al. 2003] Valsecchi G.B., Milani A., Gronchi G.F. and Chesley S.R. : 2003 Resonant returns to close approaches: Analytical theory, Astronomy \& Astrophysics, Volume 408, Number 3, pp. 1179-1196 